\title{
Quantum Transport Properties of Two-Dimensional Quantum Lattices under Synthetic Magnetic Fields
}

\author{
P.G. Wijesinghe ${ }^{1 a}$, K.A.I.L Wijewardena Gamalath ${ }^{2 b^{*}}$ \\ Department of Physics, University of Colombo, Colombo3, Sri Lanka \\ apgwijesinghe@gmail.com, bimalie@phys.cmb.ac.lk
}

\begin{abstract}
Keywords: Quantum transport properties, band structures, square lattice, triangular lattice, kagome lattice, Quantum Hall effect, Landau levels, edge states
\end{abstract}

\begin{abstract}
Motivated by recent experimental progress, we study the quantum transport properties of two-dimensional electron gases under high perpendicular magnetic fields. We use a simple tightbinding model to model the system and open-source software to simulate quantum electronic transport properties such as band structure variations and conductance-flux relationships in such systems. Dependence of quantum transport properties on two-dimensional square, triangular and kagome lattice shapes were studied adding a Gaussian noise to account for the impurities. Numerical simulations are presented to predict the emergence of physical effects related to quantum Hall effect, such as the existence of Landau levels and edge states. The kagome lattice exhibits a different band structure giving rise to a flat band, due to its trihexagonal geometry. The peak conductance value increases with decreasing lattice constant due to higher transmission probability. The transport properties vary significantly with lattice geometries, both with the lattice type and the lattice constant.
\end{abstract}

\section{Introduction}

Since electronics have become a vital element in the current context of the world transport properties of different materials have become very important. The world has already moved on from the classical regime to the quantum regime and scientists believe that the solution to the dropped pace of advancement lies in the yet fully unexplored quantum regime. One solution is to make explicit use of quantum computing which requires the use of quantum simulators and scientists are seeking different ways of building them. However, yet there are not many algorithms known in quantum information, which can make use of the quantum mechanical advantages. Another solution is to seek for different materials with different structures which have advanced transport properties. Transistors made from graphene has shown a frequency of $100 \mathrm{GHz}$, which is higher than the frequency of a silicon transistor of the same size. This presents us a hint of advancement through the seeking of new materials and structures.

Recent experimental progress in atomic, molecular and optical physics has led to the realization of artificial crystals with ultracold atoms. These systems are described by quantum lattice models. These structures can be used to mimic quantum simulations and to design and observe the transport and material properties of artificial crystals. Many of their properties can be controlled by means of external fields. To create artificial crystals, the interparticle spacing should be comparable to the de Broglie wavelength $\lambda_{d B}=\hbar \sqrt{2 \pi / m k_{B} T}$ where $m$ is the mass of the particle, $k_{B}$ is the Boltzmann constant and $T$ is the temperature. For particles as heavy as atoms, very low temperatures below $1 \mu \mathrm{K}$ and very high densities such as $10^{18}-10^{21}$ atoms $/ \mathrm{m}^{3}$ are required to reach the quantum limit [1]. Various techniques to slow down and trap atoms have been developed and the technique of Doppler cooling which slows down atoms with the use of lasers, lowering the temperatures of the particles to the order of $\mathrm{mK}$ is dominant and has evolved in to a technique called sub-Doppler cooling or laser side-band cooling which can reach a temperature limit of $\mu \mathrm{K}$. Evaporative cooling can be used to reach a temperature limit of around $500 \mathrm{pK}$ [2]. The first experimental evidence of this method was provided when the Bose-Einstein Condensate (BEC) was realized verifying Einstein's early predictions. The development of sympathetic cooling enabled the formation of degenerate Fermi gas which enabled experiments similar to that of BEC to be done with Fermions [3]. 
An alternate method to reach the highly interaction regime with ultracold atoms is to use optical lattice, series of optical potentials which can be formed by the interference of counterpropagating laser beams. The intensity of the laser beams can be controlled to form periodic potentials which can make the interactions between the particles dominate. By changing the laser configuration, we can create different Bravais lattices in any dimensions. Fig. 1 shows the atoms confined to an array of 1D potential tubes for a 2D

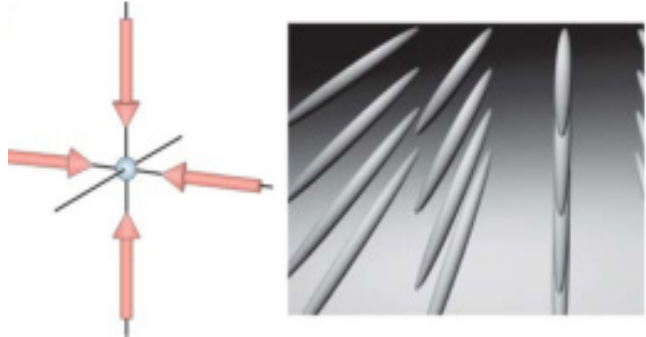

Figure 1: The atoms are confined to an array of $1 \mathrm{D}$ potential tubes for a $2 \mathrm{D}$ optical lattice optical lattice [4]. Potential depth can be controlled by controlling the power of the laser and the periodicity can be controlled by controlling the wavelengths or the relative angle between the two lasers.

Atoms trapped in optical lattices can move between potential minimas in two ways. The states whose kinetic energy is higher than the potential barrier are delocalised. Such a particle can move freely within the lattice. The atoms from the low energy bands are localised around the potential minima and their energy are too low to move between the lattice sites in a classical way. However, these atoms, can still move by tunnelling from one sit to another. In the case of high lattice potentials, the only way of moving between sites is tunnelling. Considering the lowest energy band, we could use Wannier functions which are linear combinations of Bloch functions $\psi_{k}(r) e^{-i k R_{i}}$ to expand the field operators. Wannier functions localized around $R_{i}$ which is given by:

$$
W\left(r-R_{i}\right)=\sum \psi_{k}(r) e^{-i k R_{i}} \chi(\sigma)
$$

The quantum transport properties of two-dimensional electron gases under high perpendicular magnetic fields in terms of vortex states within the framework of real-time Green functions were studied by Champel and Florence [5]. They obtain the first and third lowest order contributions to the current due to Landau-levels mixing processes, and derived the quantization of the Hall conductance.

In this paper we study artificial crystals since it is important to understand the differences and similarities between these artificial ultracold gas systems and real solid-state systems. The most prominent difference we can think of, between these two systems is, while electronic systems are connected to the external environment, ultracold gases are isolated from the environment due to the confinement and extreme low densities and temperatures it requires. Even the smallest perturbation can destroy an ultracold gas system. But at the same time, it acts as a very good quantum simulator which can mimic the dynamics of a real solid-state system. Hence these artificial lattices are very useful for experimental purposes. Quantum electronic transport properties of artificially constructed two-dimensional square, triangular and kagome lattices of 20 units x 20 units with a lattice constant of $a=1$ unit with infinite leads attached towards the $\mathrm{x}$-direction on left and right ends of the system were studied using the tight-binding model. The python package, 'Kwant' was used for the simulations with base parameters, the dimensionless quantities incorporating a discretized version of the system Hamiltonians. A perpendicular magnetic field was applied to it by using a modified hopping potential and the band structure of the leads was obtained. Applying a fixed energy of 0.2 units, the respective scattering matrix was obtained to compute the conductance values at varying magnetic flux values. Then, the Inverse magnetic flux was varied from 4 units to 30 units in 0.005 unit steps and the respective conductance values was obtained to obtain a graphical representation of the relationship between conductance and magnetic flux. The sum of probabilities of all wave functions for a given magnetic flux value was obtained to observe the effect of the applied magnetic field to the spread of wave functions. For this map, we have selected $1 / 20$ units as the magnetic flux value since at this value, the system has just started to exhibit the quantization of conductance. Then to mimic the realistic nature of materials by accounting for the impurities and imperfections of real crystals, we have added a Gaussian noise to the system and repeat the process. The transport 
properties of difference lattice geometries are discussed and numerical simulations are presented to predict the emergence of physical effects related to Quantum Hall Effect, such as existence of Landau levels and edge states.

\section{Theory}

We can study the quantum transport properties of a given region by obtaining an expression for the electric conductance $(G)$ of a conducting region with an average probability for an electron to transmit across it. Considering the 2-dimensional system given in Fig. 2, we assume that charge carriers in the leads are arranged according to a Fermi distribution,

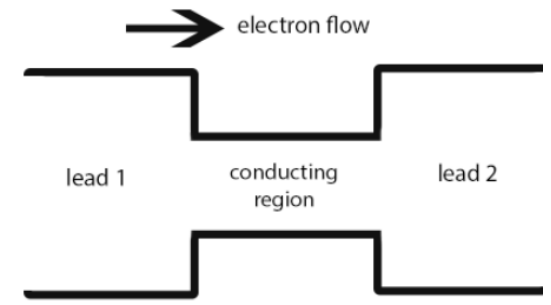

Figure 2: Conducting region positioned between two leads

$$
f_{i}(E)=\frac{1}{e^{\left(E-\mu_{i}\right) / k_{B} T}+1}
$$

where $i$ is the lead number, $E$ is the energy, $\mu_{i}$ is the chemical potential of the respective lead, $k_{B}$ is the Boltzmann constant and $T$ is the temperature of the system. The leads are similar to the conductor in one end and the other end can be considered as a large electron reservoir. These reservoirs are assumed to be large enough so that they can enter the system with zero probability of reflection. For a given energy $E$, electrons occupy $\pm k$ states where $k$ is the wave vector in the direction of propagation. $+k$ states carry positive current and $-k$ states carry negative current. Considering a cloud of positive current carrying electrons in lead 1 which is moving with a group velocity $v_{g}$ in the positive direction of propagation, the current carried by these electrons is given by,

$$
I_{1}=\sum_{k>0} v_{g} e n f_{1}(E)=\frac{e n}{\hbar} \sum_{k>0} \frac{\partial E}{\partial k} f_{1}(E)
$$

where $e$ is the electronic charge, $n$ is the carrier density in the propagating dimension. Assuming periodic boundary conditions, the sum can be written as an integral by considering the k-space geometry as follows:

$$
I_{1}=\frac{2 e}{h} \int_{E_{0}}^{\infty} f_{1}(E) d E
$$

where $E_{0}$ is the minimum energy for the respective configuration. To account for the spin degeneracy of an electron we have multiplied it by a factor of 2 . Then, the flux of charge carriers in Lead 1 moving in to the conducting region is given by:

$$
i_{1}=\frac{2 e}{h} f_{1}(E) N(E)
$$

The net electric current through the conducting region as follows:

$$
I=\frac{2 e}{h} \int_{-\infty}^{+\infty} T(E)\left(f_{1}(E)-f_{2}(E)\right) d E
$$

where $T(E)$ is the total transmission probability across the conducting region. We can define the conductance of the conducting region as $G=I / V$. Here $V=\left(\mu_{1}-\mu_{2}\right) / e$ is the applied bias between 
the two leads and the respective chemical potentials of the leads are given by $\mu_{1}=E_{F}+e V / 2$ and $\mu_{2}=E_{F}-e V / 2$ where $E_{F}$ is the Fermi energy [6]. The conductance:

$$
G=\frac{2 e^{2}}{h V} \int_{-\infty}^{+\infty} T(E)\left(f_{1}(E)-f_{2}(E)\right) d E
$$

The Hubbard model is a good approximation for particles in a periodic potential at sufficiently low temperatures that all the particles are in the lowest Bloch band, as long as any long-range interactions between the particles can be ignored. The Hubbard Hamiltonian is given by:

$$
H=-\sum_{i, j, \sigma, \sigma^{\prime}} t_{i, j, \sigma, \sigma^{\prime}}\left(c_{i \sigma}^{\dagger} c_{j \sigma}+h . c\right)+U \sum_{i} n_{i \uparrow} n_{i \downarrow}-\mu \sum_{i} n_{i}
$$

where $i, j$ are the sites in the lattice, $c^{\dagger}$ is the creation operator, $c$ is the annihilation operator, $\mathrm{U}$ is the near particle interaction term and $\mu$ is the chemical potential and h.c. is the Hermitian conjugation. Here, it is assumed that long-range interactions are negligible and only the nearest-neighbour tunnelling contributes to the Hamiltonian and interactions only inside one lattice site. The above description can be further modified by neglecting the interaction term. It is called the tight-binding Hamiltonian and will be used to simulate lattices.

$$
H^{T B}=-\sum_{i, j, \sigma, \sigma^{\prime}} t_{i, j, \sigma, \sigma^{\prime}}\left(c_{i \sigma}^{\dagger} c_{j \sigma}+h . c\right)
$$

A gauge theory is any field theory, in which the Hamiltonian is invariant under a group of local transformations. The gauge refers to the redundant degrees of freedom used to describe the field in the Hamiltonian that can be changed, leaving the physics of the system unchanged [1].

The Schrodinger equation for a particle with charge $q$ in an electromagnetic field, given by the vector potential $\boldsymbol{A}$ and the scalar potential $\phi$ is:

$$
\left[\frac{\hbar^{2}}{2 m}(-i \nabla-q \boldsymbol{A})^{2}+q \phi\right] \psi(\boldsymbol{r}, t)=i \frac{\partial \psi(\boldsymbol{r}, t)}{\partial t}
$$

Let us consider the transformation:

$$
\boldsymbol{A}^{\prime}=\boldsymbol{A}+\nabla^{\prime} \Lambda ; \quad \phi^{\prime}=\phi-\frac{\partial^{\prime} \Lambda}{\partial t}
$$

Although, the Maxwell equations do not change under this transformation, Schrodinger equation changes and the wave function transforms accordingly as follows:

$$
\psi^{\prime}(\boldsymbol{r}, t)=e^{i q \Lambda} \psi(\boldsymbol{r}, t)
$$

The Schrodinger equation under the new transformation:

$$
\left[\frac{\hbar^{2}}{2 m}\left(-i \nabla-q \boldsymbol{A}^{\prime}\right)^{2}+q \phi^{\prime}\right] \psi^{\prime}(\boldsymbol{r}, t)=i \frac{\partial \psi^{\prime}(\boldsymbol{r}, t)}{\partial t}
$$

Solving Eq. 10 and Eq.12, we can arrive at the transformations for the differential operators:

$$
\nabla^{\prime}=\nabla-q A ; \quad \frac{\partial^{\prime}}{\partial t}=\frac{\partial}{\partial t}+i q \phi
$$


By substituting the modified operators in Eq. 11, we obtain the Schrodinger equation which is gauge invariant under the above transformation.

The quantum Hall effect is a quantum-mechanical version of the Hall effect, observed in 2dimensional electron gases subjected to low temperatures and strong magnetic fields, in which the Hall conductance $\sigma$ experiences quantum Hall transitions to take on the quantized values. Quantum Hall effect arises in two different forms, namely, integer quantum Hall effect (IQHE) and fractional quantum Hall effect (FQHE). However, we restrict only to IQHE. The Hall conductance can be found by the relationship:

$$
\sigma=\frac{I}{V_{\text {Hall }}}=\frac{v e^{2}}{h}
$$

where $v_{v}$ is the filling factor of the respective system, $e$ is the electronic charge and $h$ is the Planck constant [7].

For free electrons whose mass and momentum are given by $m$ and $\boldsymbol{p}$ respectively, confined to a $2 \mathrm{D}$ plane, we can express the Hamiltonian as:

$$
H=\frac{1}{2 m}(\boldsymbol{p}-e \boldsymbol{A})^{2}+V
$$

where $\boldsymbol{A}$ is the vector potential which defines the magnetic field $\boldsymbol{B}=\nabla \times \boldsymbol{A}$ acting perpendicular to the 2D plane, and $V$ is the scalar potential. Solving the TISE for the above Hamiltonian, we can arrive at the energy eigenvalues given by $E_{n}=\hbar \omega_{c}(n+1 / 2)$ given by the cyclotron frequency $\omega_{c}=e B / m$. This corresponds to the Hamiltonian of a harmonic oscillator. These orbitals are known as Landau levels. At weak magnetic fields, their existence gives rise to "quantum oscillations" whereas at strong magnetic fields, these levels become degenerate. For electrons in a 2D lattice, we can express the Hamiltonian using the tight-binding model given in Eq. 9 as $H^{T B}=\sum_{i j} t_{i j} c_{i}^{\dagger} c_{j}$ where the hopping potential $t_{i j}$ between site $i$ and $j$ is given by:

$$
t_{i j}=t e^{-i \varphi_{i j}} ; \quad \varphi_{i j}=\frac{e}{\hbar} \int_{i}^{j} \boldsymbol{A}(\boldsymbol{r}) \cdot d \boldsymbol{r}
$$

$t$ is the hopping potential between two sites when the corresponding magnetic field strength is zero.

To solve the Schrodinger equation corresponding to the Hamiltonian given by Eq.13, we follow a numerical approach. Considering the system given in Fig. 2, we assume both leads to have the same $N$ number of occupied transversal modes at a given fixed energy. Once the solutions corresponding to all these modes are obtained, the scattering $2 N \times 2 N$ matrix $S$ connect, the column vector of outgoing complex wave amplitudes $c_{\text {out }}$ to the column vector of incoming complex wave amplitudes $c_{\text {in }}$ as $c_{\text {out }}=S c_{\text {in }}$ and is given by:

$$
S=\left(\begin{array}{ll}
r_{1} & t_{2} \\
t_{1} & r_{2}
\end{array}\right)
$$

Here $t_{i}$ and $r_{i}$ are the transmission and reflection amplitudes of electrons originating in the Lead $i(i=1,2)$. We can use this scattering matrix to obtain the total transmission probability is given by $T(E)=\sum_{i, j}\left|t_{i j}\right|^{2}$ 
Time independent Hamiltonian for one-dimensional system with lattice constant $a$ can be written using the method of finite differences to approximate as [8]:

$$
H \psi(x)=\frac{\hbar}{2 m} \frac{d^{2} \psi(x)}{d x^{2}}=\frac{\hbar}{2 m a^{2}}\left(\frac{2 \psi(x)-\psi(x-a)-\psi(x+a)}{a^{2}}\right)=E \psi(x)
$$

Comparing the terms of the above equation, we can read $2 \hbar /\left(2 m a^{2}\right)$ as the onsite energy term and $-\hbar / m a^{2}$ as the hopping energy. Extending this structure to 2- dimensions, we can obtain onsite energy as $4 t$ and hopping energy as $-t$ where $t=\hbar / 2 m a^{2}$ which has the dimensions of energy.

We embed a magnetic field $\boldsymbol{B}$ which accounts for a vector potential $\boldsymbol{A}$. For a constant magnetic field applied in the direction perpendicular to the lattice (z-direction), preventing the translational symmetry through the x direction, we can choose $\boldsymbol{A}=-B y \hat{\boldsymbol{x}}$. To embed this vector potential in the tight binding model describing the square lattice is done by using Peierls substitution to replace the hopping potential as [9]:

$$
t_{i j}{ }^{\prime}=t_{i j} \exp \left(i e / \hbar \int_{i}^{j} A(x) \cdot d r\right)
$$

We can do this as follows where $i$ and $j$ are lattice sites, $t_{i j}{ }^{\prime}$ is the modified hopping potential. In order to compute the transport properties, we use the open-source python package called Kwant [10].

\section{Square Lattice}

We use Kwant package [9] to create a 2D square lattice of dimensions $20 \times 20$ units with a lattice constant of 1 unit. This lattice acts as the conducting region of the system. Infinite leads are attached to the left and right sides of the lattice, preserving the translational symmetry along the $\mathrm{x}$ direction (Fig. 2). These leads act as the reservoirs of charge carriers. By calculating the transmission of charge carriers through the conducting region, we can obtain the scattering matrix of the system which can be

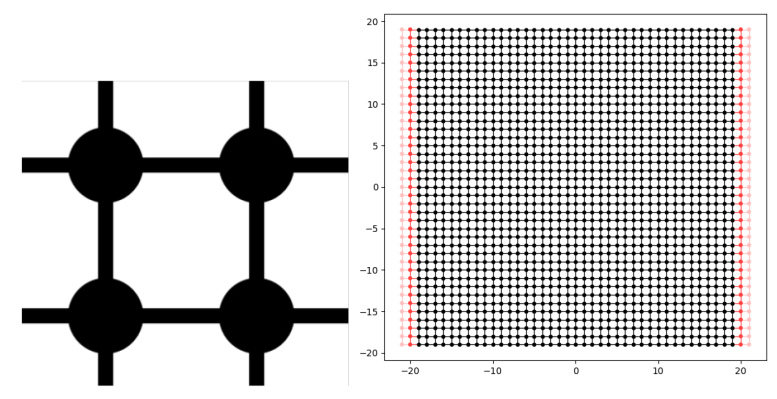

Figure 3: Non-primitive unit cell (left) of the system and the constructed 2D square lattice with leads (red) attached (right) used to compute the transport parameters using the numerical approach. When a magnetic field is embedded, the hopping potential of the system is modified. For a square lattice with lattice constant $a$, magnetic flux through a unit cell is $\phi=\boldsymbol{B} \cdot \boldsymbol{A}=B a^{2}$ and flux quantum is $\phi_{0}=h / e$. The onsite potential for the lattice is $4 t$. Then the hopping potential is:

$$
t_{i j}{ }^{\prime}=t_{i j} \exp \left(i \frac{2 \pi \phi}{\phi_{0}}\left(y_{i}+y_{j}\right) \frac{\left(x_{i}-x_{j}\right)}{2 a^{2}}\right)
$$


We can obtain the band structure of the leads, that is the energies of the propagating plane waves in the leads as a function of momentum. This band structure contains information about the number of modes, their momenta and velocities. To preserve the generality, we use the numerical parameters relative to their base parameters i.e. magnetic flux $\phi / \phi_{0}$, energy $E / E_{0}$ and conductance $G / G_{0}$ where $\phi_{0}=h / e, \quad E_{0}=\hbar / 2 m a^{2} \quad$ and $G_{0}=2 e^{2} / h$. One base unit of energy was

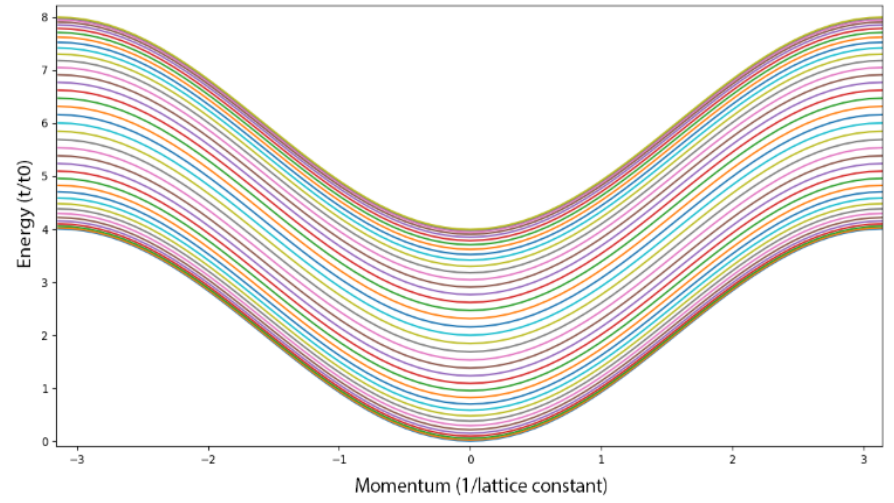

Figure 4: Band structure of the left infinite lead of the $2 \mathrm{D}$ square lattice chosen for convenience. The band structure of the left infinite lead is presented in Fig. 4. This shows a cosine pattern.

An initial fixed energy of 0.2 units was fed into the system to obtain the respective scattering matrix using the Kwant package. Then, by varying the magnetic flux from 1/30 units to $1 / 4$ units, graphical representation of the relationship between magnetic flux and conductance was obtained. This is shown in Fig. 5(a). The conductance of the system shows a step-wise decrease in value with increasing magnetic flux. These steps (plateaus) occur at the Landau levels of the system. The probabilities of all possible wave functions of the system was obtained to observe the way the system changes with the applied magnetic field and presented in Fig. 5(b) for applied magnetics flux of 0.05 units. The wave function is localized towards an edge showing the emergence of edge states of the system.

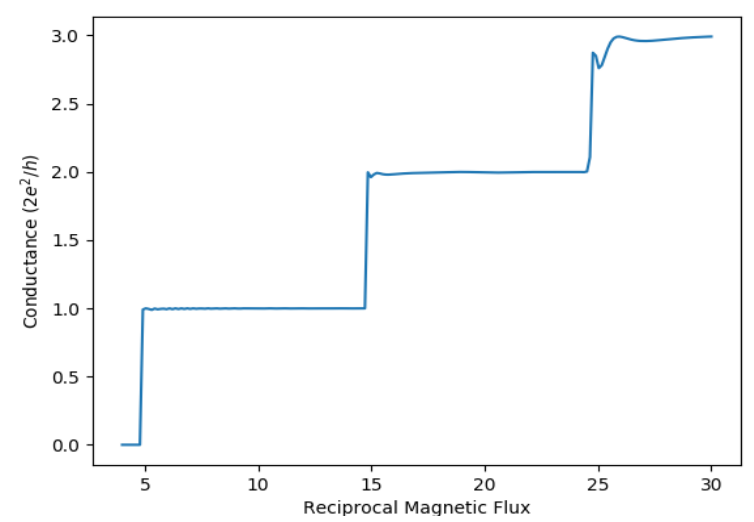

Figure 5(a): Conductance and the reciprocal of magnetic flux for a $2 \mathrm{D}$ square lattice

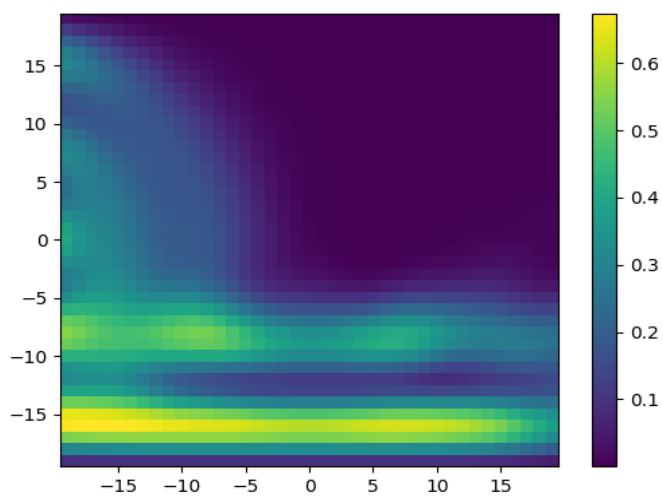

Figure 5(b): Sum of all wave function probabilities of the system with applied magnetic flux of 0.05 units

The real material structures show disorder due to the imperfections or impurities in crystals. This disorder is a fundamental source of non-ballistic transport. Thus, we include a disorder in our model using a Gaussian disorder model (GDM), a distribution centred at zero, to obtain a more realistic result. The above process was repeated by adding a Gaussian noise to the onsite potential to account for the possible impurities and imperfections of the crystal. The relationship between the embedded magnetic flux and the conductance of the system and of probabilities of all possible wave functions of the system for applied magnetic flux of 0.05 units with the Gaussian noise is presented in Fig. $6(a)$ and $6(\mathrm{~b})$ respectively. The conductance of the system still shows a step-wise decrease with increasing magnetic flux regardless of the added noise and theses steps occur at the Landau levels of the system. The wave function is also still localized towards an edge showing the emergence of edge states of the system regardless of the added noise although the noise is incorporated in the graphs. 


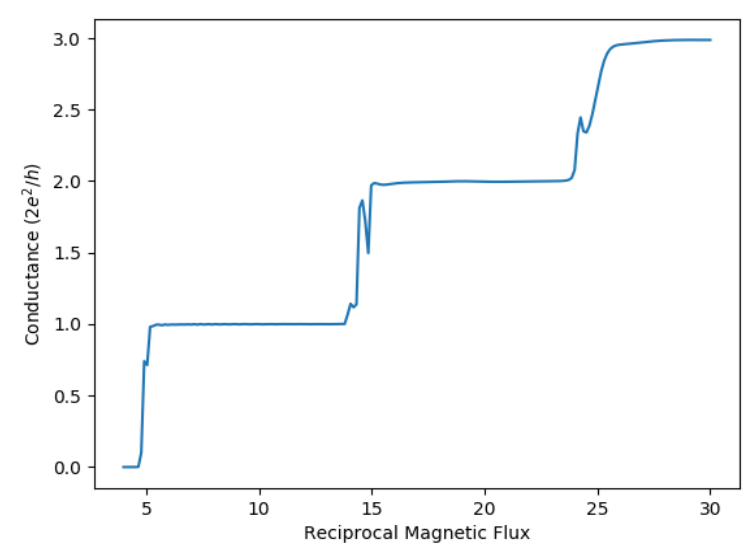

Figure 6(a): Conductance and the reciprocal of magnetic flux for a 2D square lattice with Gaussian noise

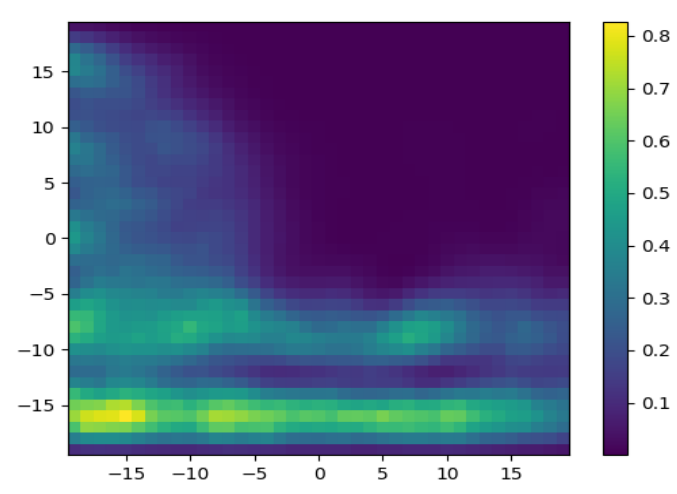

Figure 6(b): Sum of all wave function probabilities of the system with applied magnetic flux of 0.05 units with Gaussian noise

The lattice constant of the system was varied from 0.5 units to 2 units in 0.25 unit steps. In each of these steps, the band structure and the relationship between conductance and magnetic flux was obtained. The band structure of infinite lead was obtained for lattice constants $a=0.5, a=1.0$, $a=1.5$ and $a=2$ in 0.25 steps and conductance with magnetic flux on square lattice shapes are shown in Fig. 7(a) and 7(b) respectively. Energy spacing increases with increasing lattice constant, resulting in a lower density of states. The conductance with magnetic flux decreases with increase lattice constant. The peak values of conductance have increased with decreasing values of lattice constants. This could be explained by the band structure picture because with decreasing lattice constant, the density of states increases. Hence, the conductance should increase because of the increased transmission probability $(T(E))$. The number of conduction plateaus have decreased with increasing lattice constant. This is due to the Landau level degeneracy and spacing increasing with increased lattice constant. Hence, for a larger lattice constant, less Landau levels are occupied. As the number of Landau levels determines the number of edge channels, we have less edge channels for increased lattice constants.
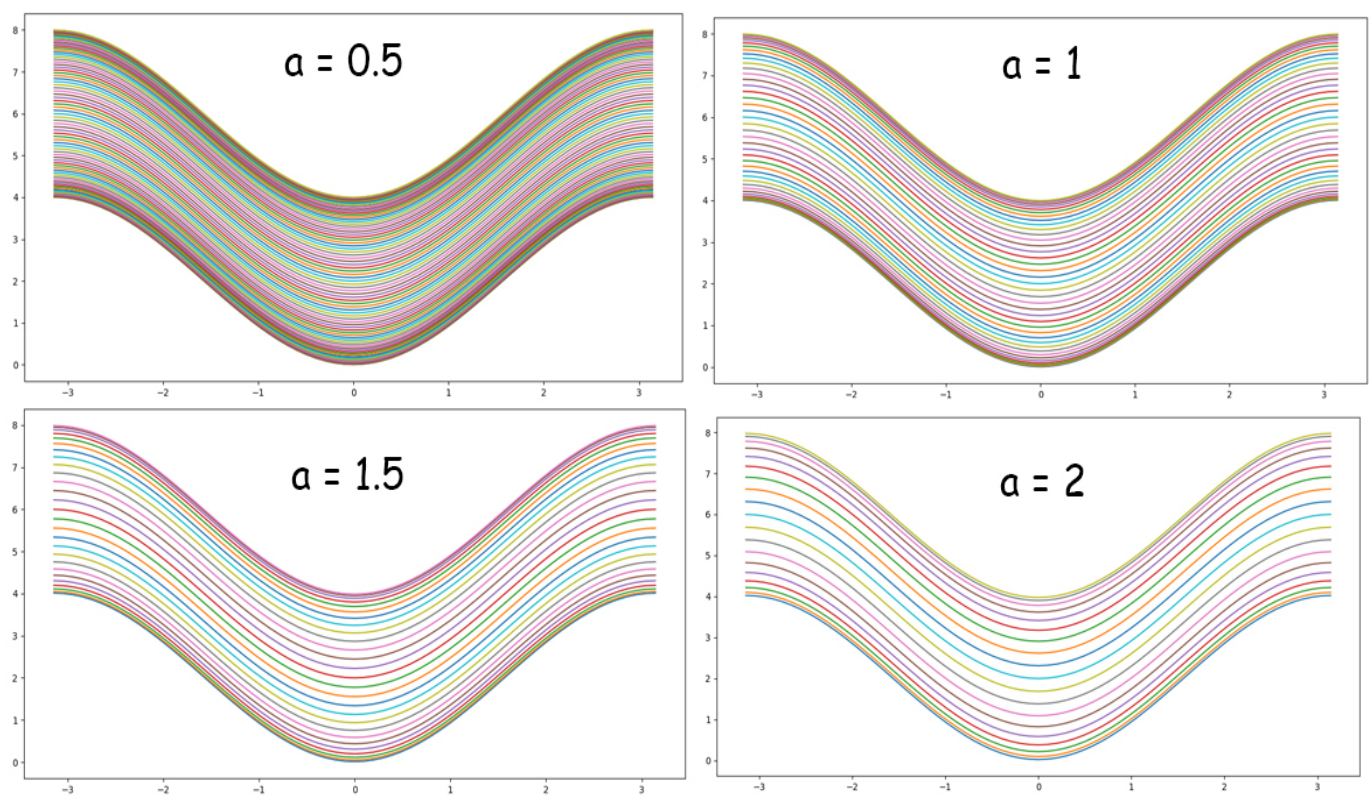

Figure 7(a): Band structures on different lattice constants in a 2D square lattice 


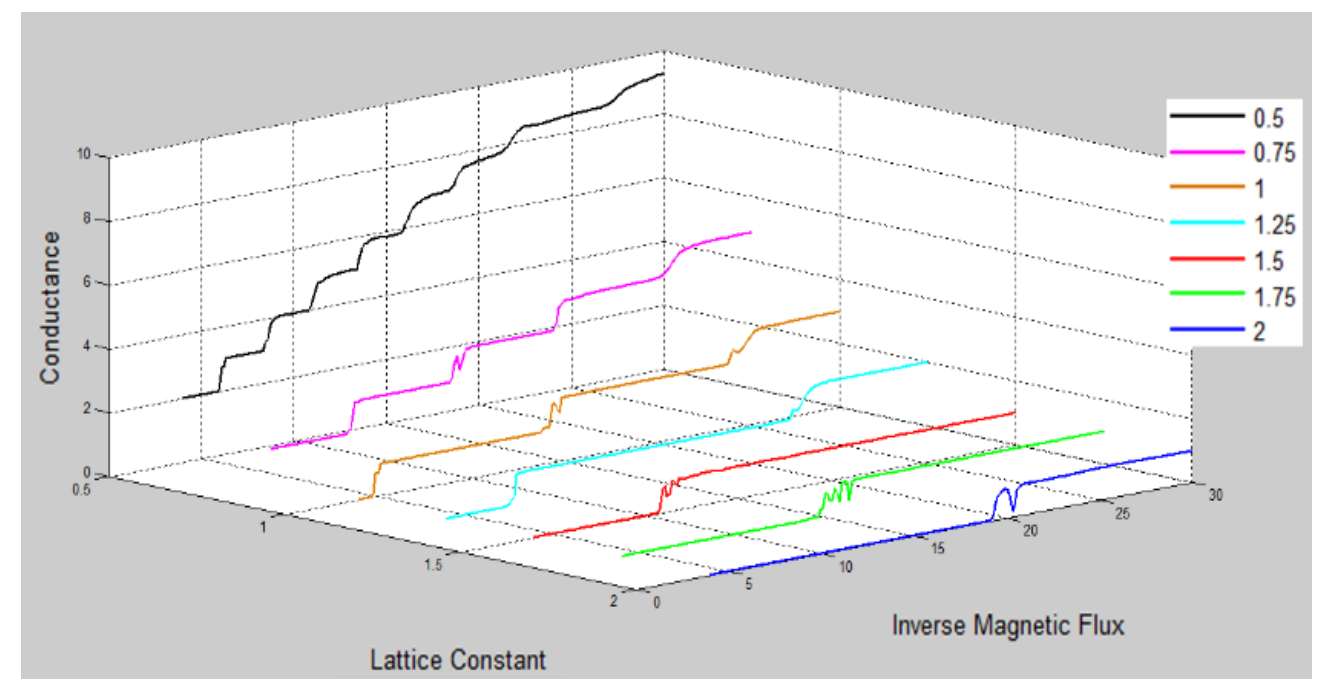

Figure 7(b): Conductance and magnetic flux for different lattice constants in a 2D square lattice

\section{Lattice Triangular}

We use the Kwant package to create a $2 \mathrm{D}$ triangular lattice of dimensions $20 \times 20$ units with a lattice constant of 1 unit and infinite leads were attached to the sides of the lattice, preserving the translational symmetry along the $x$ direction as shown as shown in Fig.8. For a triangular lattice with lattice constant $a$, magnetic flux through a unit cell is $\phi=B \times a / 2 \times \sqrt{3} a / 2$ and flux quantum is $\phi_{0}=h / e$. The onsite potential for the lattice is $4 t$. The hopping potential is:

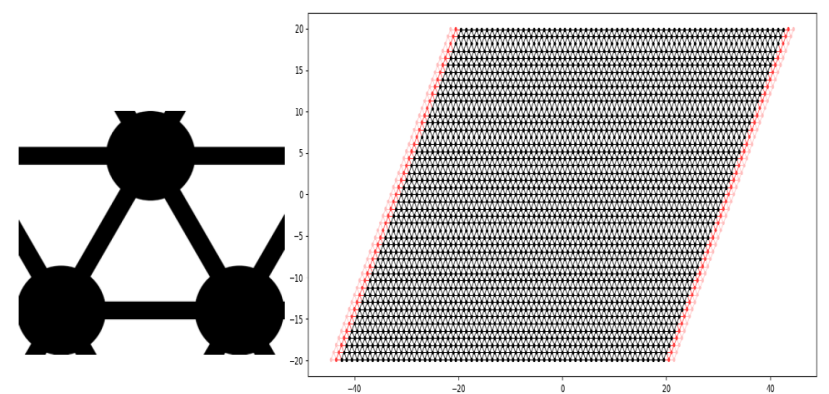

Figure 8: Non-primitive unit cell (left) of the system and the constructed 2D triangular lattice with leads (red) attached (right)

$$
t_{i j}{ }^{\prime}=t_{i j} \exp \left(i \frac{2 \pi \phi}{\phi_{0}}\left(y_{i}+y_{j}\right) \frac{2\left(x_{i}-x_{j}\right)}{\sqrt{3} a^{2}}\right)
$$

Using the numerical parameters relative to their base parameters we obtain the band structure of the left infinite lead leads. Here also, the one base unit of energy was chosen for convenience. The band structure is shown in Fig. 9 and it follows a cosine pattern. The energy bands are more spread out than for the square lattice resulting in a lower density of states.

A Gaussian noise was added to the system for the real behaviour of the system. An initial fixed energy of 0.2 units to the system and obtain the respective scattering matrix using the Kwant package. By varying the magnetic flux from $1 / 30$ units to $1 / 4$ units the obtained a graphical representation of the relationship between magnetic flux and conductance is shown in fig. 10(a). The conductance of the system shows a step-wise increase with decreasing

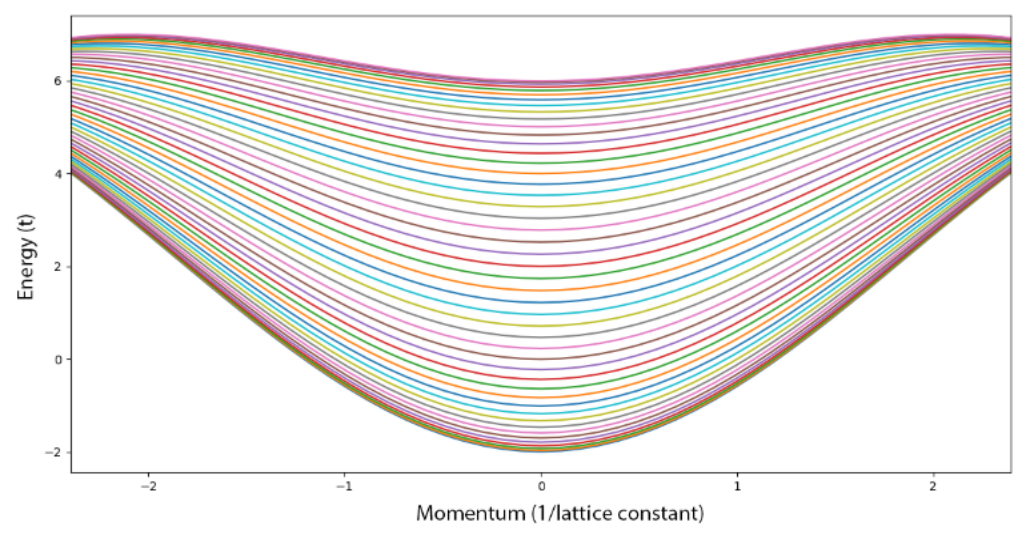

Figure 9: Band structure of the left infinite lead of the 2D triangular lattice 
magnetic flux showing the Landau levels of the system. The probabilities of all possible wave functions of the system is presented in Fig. 10(b). The wave function is localized towards an edge showing the emergence of edge states of the system.

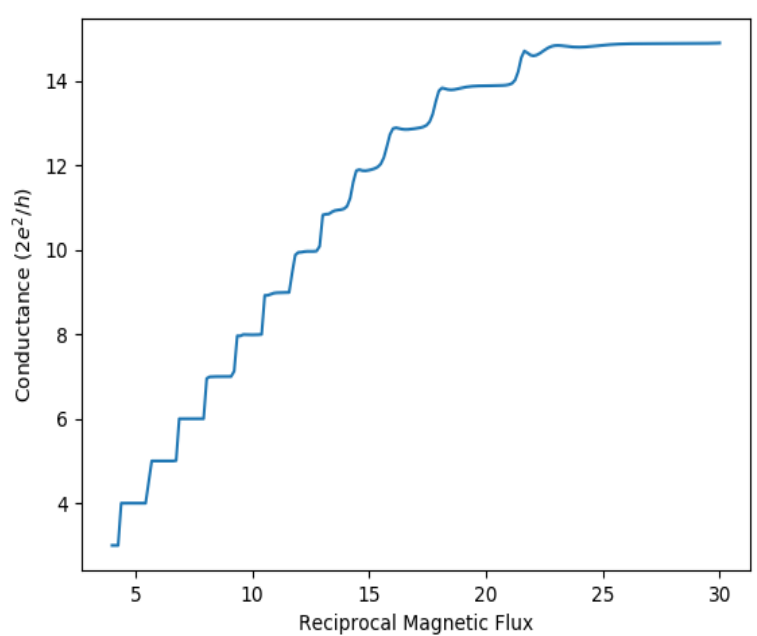

Figure 10(a): Relationship between conductance and the reciprocal of magnetic flux for a $2 \mathrm{D}$ triangular lattice with Gaussian noise

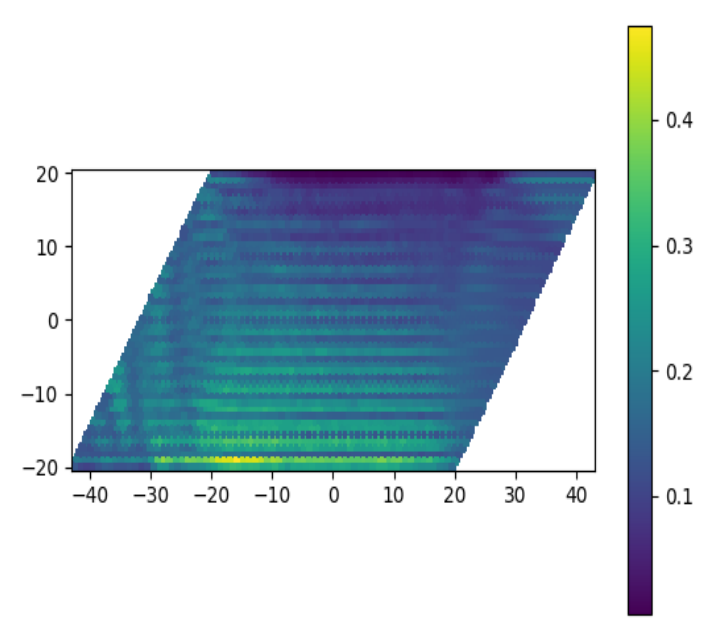

Figure 10(b): Sum of all wave function probabilities of 2D triangular lattice with applied magnetic flux of 0.05 units with Gaussian noise

\section{D Kagome Lattice}

The Kwant package was used to create a 2D kagone lattice of dimensions 20 x 20 units with a lattice constant of 1 unit and infinite leads attached to sides of the lattice, preserving the translational symmetry along the $x$ direction as shown in Fig.11. Hopping potential was modified to account for the kagome lattice. The magnetic flux through a unit cell of lattice constant $a$ is $\phi=B \times 12 \times \frac{a}{2} \times \frac{\sqrt{3} a}{2}$ and the flux
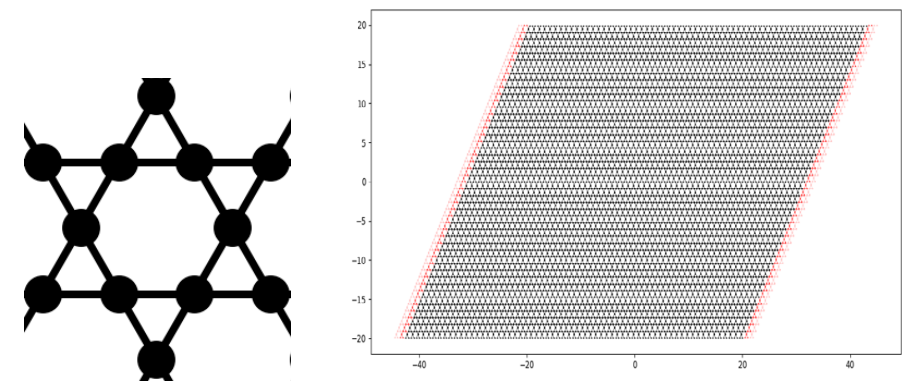

Figure 11: Non-primitive unit cell (left) of the system and the constructed 2D kagome lattice with leads (red) attached (right) quantum is $\phi_{0}=h / e$. The onsite potential for the lattice is $4 t$. The hopping potential is:

$$
t_{i j}{ }^{\prime}=t_{i j} \exp \left(i \frac{2 \pi \phi}{\phi_{0}}\left(y_{i}+y_{j}\right) \frac{\left(x_{i}-x_{j}\right)}{3 \sqrt{3} a^{2}}\right)
$$

The band structure of the left infinite lead was obtained relative to their base parameters and presented in Fig. 12. A flat band was obtained for this lattice. This can have various physical interpretations. In the momentum space, looking at the dispersion relation, a flat band means the effective mass of the particle is infinite, hence, it cannot move and therefore not dispersive. In the real space, it means the hopping of the particle between different regions is

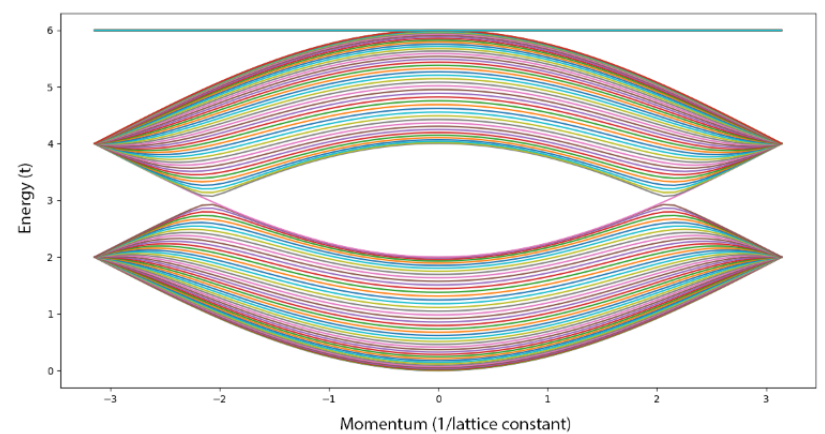

Figure 12: Band structure of the left infinite lead of the 2D kagome lattice effectively turned off, such that the particle is localized. 
A Gaussian noise was added to the system and an initial fixed energy of 0.2 units was added to the system to obtain the respective scattering matrix using the Kwant package. Then, by varying the magnetic flux from $1 / 30$ units to $1 / 4$ units, the relationship between the embedded magnetic flux and the conductance of the system was obtained and presented in Fig 13(a). The conductance of the system shows a step-wise increase with decreasing magnetic flux. These steps occur at the Landau levels of the system. The probabilities of all possible wave functions of the system obtained is presented in Fig. 13(b). The wave function is localized towards an edge showing the emergence of edge states of the system. The conductance of the system shows a stepwise decrease with increasing magnetic field, as increasing the magnetic field reduces scattering due to the formation of quantum Hall edge channels. and the increase occur with the largest number of steps. reduces scattering due to the formation of quantum Hall edge channels.

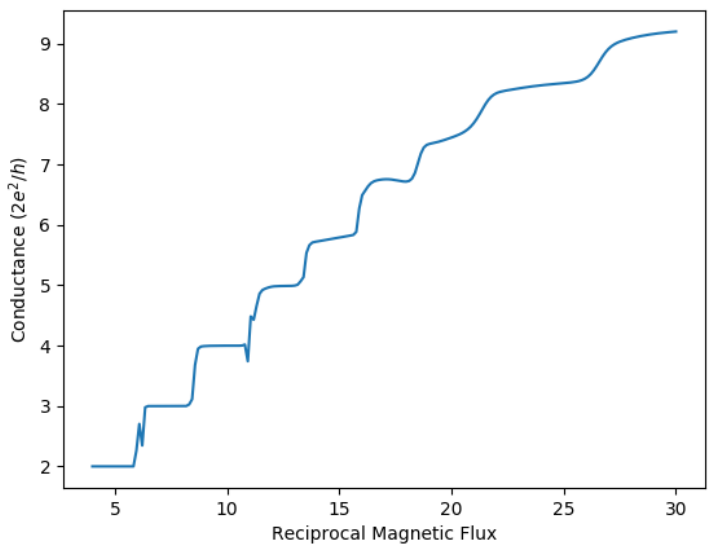

Figure 13(a): The conductance and the reciprocal of magnetic flux for a 2D kagome lattice with Gaussian noise

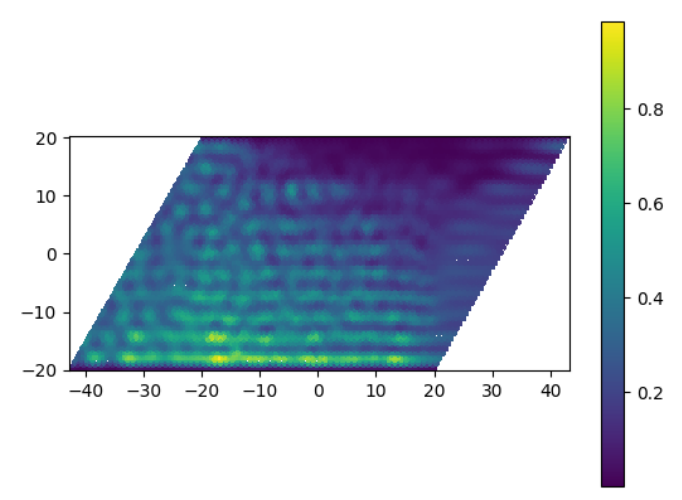

Figure 13(b): Sum of all wave function probabilities of 2D kagome lattice with applied magnetic flux of 0.05 units with Gaussian noise

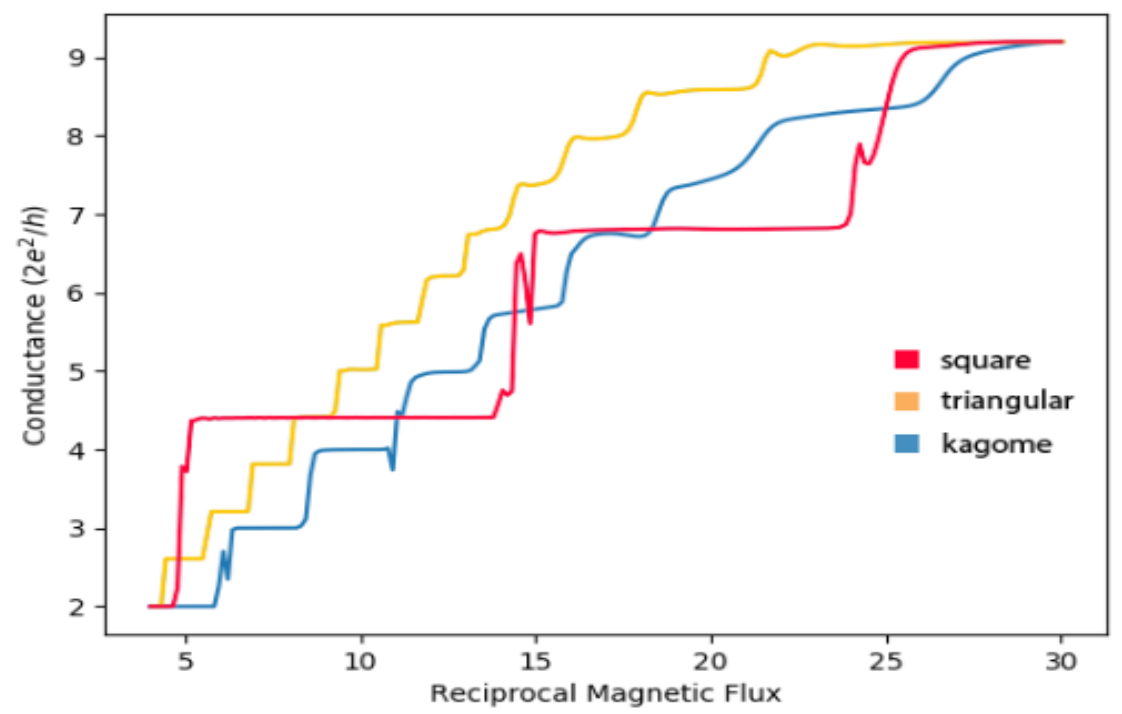

Figure 14: The conductance and the reciprocal of magnetic flux for a 2D, square, triangular and kagome lattice with Gaussian noise

The conductance and magnetic flux for square, triangular and kagome lattice shapes are shown in Fig. 14. The conductance increases with reciprocal magnetic flux in three steps for the square lattice while kagome lattice has higher conductance when the reciprocal magnetic flux is higher than 10 units. 


\section{Discussion}

For square triangular and kagome artificial lattices, the band structure shows a cosine pattern and energy is distributed in discrete values. This discreteness can be considered as evidence for the existence of Landau levels of the system when analysed through the quantum regime. The conductance of the system shows a stepwise decrease with increasing magnetic field, because increasing the magnetic field reduces scattering due to the formation of quantum Hall edge channels. Hence, we see the conductance quantization. In low magnetic field regimes, conductance shows a nearly constant variation and only when the magnetic flux is increased above $1 / 25$ units, the system exhibits quantum Hall effect and the conductance starts to drop in steps, specifically in multiples of $2 e^{2} / h$. These steps (plateaus) occur at the Landau levels of the system. The density plots showed that as a result of the applied magnetic field, the wave functions moved to an edge of the system giving evidence for the formation of edge states in the system. The three different lattice geometries have resulted in varying step sizes as these step sizes depend on the filling factor of the lattice. However, it can be seen that this does not happen uniformly. There are always some values of the magnetic flux, where the wave function starts to spread again into the whole system, although it is already localized to an edge. One possible explanation for this fact is that for these specific magnetic flux values, system exhibits Landau levels close to the given energy. The finite disorder broadens the Landau level, giving rise to a bulk conductance. We can observe this clearly when we correlate the band structure plots with the wave function plots. The conductance goes down with increasing magnetic field due to the Landau level degeneracy and spacing increases with magnetic field. Hence, for a larger field, less Landau levels are occupied. As the number of Landau levels determines also the number of edge channels, we have less edge channels for increasing magnetic fields.

Unlike square and triangular lattice types, kagome lattice gives rise to a flat band for the left end lead. In this case, in the momentum space the effective mass of the particle is infinite and cannot be moved and therefore not dispersive. In the real space, the hopping of the particle between different regions is effectively turned off, so that the particle is localized. The energy spacing increases with increasing lattice constant decreasing the density of states. The peak values of conductance increase with decreasing lattice constants because of the increased transmission probability. The number of conduction plateaus have decreased with increasing lattice constant. This is due to the Landau level degeneracy and spacing increasing with increased lattice constant. Hence, for a larger lattice constant, less Landau levels are occupied. As the number of Landau levels determines the number of edge channels, and there are less edge channels for increasing lattice constants.

\section{Conclusions}

The focus of this paper is in gauge fields that can be efficiently simulated in ultracold artificial atomic systems. Quantum transport properties of two-dimensional electron systems under high perpendicular magnetic fields were studied using the tight-binding model. The dependence of quantum transport properties studied for 2D square, triangular and kagome lattices, with an equal lattice constant of 1 unit gave band structures for each configuration a cosine pattern and a discrete energy distribution which showed the existence of Landau levels of such systems. However, the band structure of kagome lattice deviated from the other two lattices by exhibiting a flat band. This may be due to these particles been trapped in a hexagon-ring state which exhibits a destructive interference induced self-localization phenomenon on each hexagon. The conductance-flux relationships obtained for each lattice type showed the quantization of conductance at high magnetic fields. These quantizations occurred at their respective Landau levels as expected. The density plots obtained for each lattice case, showed the emergence of edge states in the magnetic flux values and exhibited conductance quantization. With decreasing lattice constant of a system with a fixed dimension, the number of plateaus increases due to the higher density of states. Therefore, the peak conductance value increases with decreasing lattice constant due to higher transmission probability. The transport properties vary significantly with lattice geometries, both with the lattice type and the lattice constant and ultracold atomic systems are a good candidate for the quantum simulators. It is possible to 
simulate a wide range of phenomena using synthetic fields in these ultracold atomic systems. Although the creation of gauge fields in a laboratory is still a challenge, a very fast progress in this field is being done.

\section{References}

[1] A. Kubasiak, Ultracold gases in non-Abelian synthetic gauge fields, Ph.D. Thesis, Institute of Photonic Sciences, Spain, 2011.

[2] C.N. Cohen-Tannoudji, W.D. Phillips, New Mechanisms for Laser Cooling, Physics Today. 43 (10) (1990) 33-40.

[3] F. Dalvofo et al., Theory of Bose-Einstein condensation in trapped gases, Rev. Mod. Phys. 71(3) (1999) 463-512.

[4] I. Bloch, Ultracold quantum gases in optical lattices, Nature Physics. 1 (2005) 23-30.

[5] T. Champel, S. Florens, Quantum transport properties of two-dimensional electron gases under high magnetic fields, Phys. Rev. B 75(24) (2007) 245326.

[6] S. Datta, Electronic Transport in Mesoscopic Systems, Indiana: Cambridge University Press, 1995.

[7] P. Soltan-Panahi et al., Quantum phase transition to unconventional multi-orbital superfluidity in optical lattices, Nature Physics. 8 (2012) 71-75.

[8] L. Beirão da Veiga, L. Lopez, G. Vacca, Mimetic Finite Difference methods for Hamiltonian wave equations in 2D, Computers \& Mathematics with Applications. 74(5) (2017) 1123-1141.

[9] K. Jiménez-García et al., Peierls Substitution in an Engineered Lattice Potential, Phys. Rev. Lett. 108 (2012) 225303.

[10] C.W. Groth et al., Kwant: a software package for quantum transport, New Journal of Phys. 16(6) (2014) 063065. 